\title{
PREFACE -ISPRS and GEO workshop on Geospatially-enabled SDGs Monitoring for the 2030 Agenda
}

\author{
Hao $\mathrm{Wu}^{1}$, Xiaoguang Zhou ${ }^{2}$, Dongyang $\mathrm{Hou}^{2}$ \\ ${ }^{1}$ National Geomatics Center of China, Beijing, China \\ ${ }^{2}$ School of Geosciences and Info-Physics, Central South University, Changsha, China
}

In September 2015, the United Nations adopted "Transforming Our World: the 2030 Agenda for Sustainable Development" as a new ambitious global development plan to end extreme poverty, fight inequality and injustice, and combat climate change. Comprising 17 Sustainable Development Goals (SDGs) with 169 associated targets, the agenda recognizes the complex and diverse challenges that the world of today faces. It has been proposed by the United Nations Committee of Experts on Global Geospatial Information Management (UN-GGIM) that it is impossible to measure and monitor the SDGs without the use of accurate, reliable and up-to-date geospatial data. Therefore, establishing a dynamic SDGs monitoring mechanism based on geospatial data is a critical issue for international communities.

This ISPRS and GEO workshop aimed at providing a focus forum to discuss the opportunities and challenges of geospatial-enabled SDGs monitoring (GI4SDGs), to present the recent R\&D progress on relevant topics (including global mapping and collaborative crowd-sourced cloud mapping), and to explore future collaborations. The workshop was organized in conjunction with ISPRS IC WG IV/III (Global mapping: updating, verification and interoperability), ISPRS WG IV/4 (Collaborative Crowdsourced Cloud Mapping), GEO Community Activity (Land Cover and Land Cover Change), Central South University and National Geomatics Center of China.

The workshop received 26 submissions, which were all reviewed by the scientific and organizing committees. According to the review results, 17 full papers were accepted and published in ISPRS Archives. There were 28 oral presentations including invited presentations in the workshop. We hope these contributions would trigger new interesting ideas or solutions for monitoring the SDGs with geospatial data in the near future.

At last, we cordially thank all the authors/speakers for their contributions and all reviewers for their constructive and valuable comments. 